Western University

Scholarship@Western

Medical Biophysics Publications

Medical Biophysics Department

$1-1-2015$

\title{
The effects of nonvascularized versus vascularized bone grafting on calvarial defect healing.
}

Stephanie M Power

Damir B Matic

David W Holdsworth

Follow this and additional works at: https://ir.lib.uwo.ca/biophysicspub

Part of the Medical Biophysics Commons

Citation of this paper:

Power, Stephanie M; Matic, Damir B; and Holdsworth, David W, "The effects of nonvascularized versus vascularized bone grafting on calvarial defect healing." (2015). Medical Biophysics Publications. 47.

https://ir.lib.uwo.ca/biophysicspub/47 


\title{
The Effects of Nonvascularized Versus Vascularized Bone Grafting on Calvarial Defect Healing
}

\author{
Stephanie M. Power, MD, MSc, * Damir B. Matic, MD, MSc, *†t and David W. Holdsworth, PhD\$//
}

\begin{abstract}
It remains unknown whether bone graft vascularity influences calvarial healing. The purposes of this study were (1) to develop a model to study nonvascularized and vascularized calvarial grafts as well as (2) to compare effects of bone graft vascularity on calvarial healing. Bilateral calvarial defects were created in 26 Wistar rats. The defects were left empty within 1 parietal region. On the contralateral side, the defects were partially closed with native parietal bone (control group, $\mathrm{n}=6$ ), nonvascularized $(\mathrm{N}-\mathrm{V}$, $\mathrm{n}=10$ ), or vascularized bone grafts (VAS, $\mathrm{n}=10$ ). The vascularized grafts were supplied by perforating dural arterioles. Bone mineralization and healing patterns from serial microcomputed tomographic scans were compared within and across the groups using parametric and nonparametric tests. Differences in bone mineral content across sides were significant between the groups at weeks $6(P=0.016)$ and $12(P=0.025)$. Bone formation was greater within both the control and VAS groups versus the N-V group at weeks 6 and 12 $(P<0.05)$. Healing patterns differed between the groups $(P<0.05)$, progressing through islands of new bone formation within the control and VAS groups while limited to defect margins on the N-V graft side. In conclusion, a bilateral calvarial defect model was established to study bone graft vascularity. Bone quantity and healing patterns differed in the presence of the nonvascularized versus vascularized grafts. Although the calvarial defect model is often applied within the plastic surgery literature to study bone substitutes, greater understanding of basic mechanisms influencing calvarial healing is first needed to avoid confounding results.
\end{abstract}

Key Words: Calvarial defect, bone healing, bone graft, vascularized, nonvascularized

(J Craniofac Surg 2015;26: 290-295)

From the *Division of Plastic and Reconstructive Surgery, and $†$ Paediatric Surgery, Departments of $\ddagger$ Paediatrics, §Medical Biophysics, and $\|$ Surgery, University of Western Ontario, London, Ontario, Canada.

Received April 7, 2014.

Accepted for publication July 23, 2014.

Address correspondence and reprint requests to Stephanie M. Power, MD, MSc, 199 Avenue Road, Toronto, Ontario, Canada, M5R 2J3 or London Health Sciences Center, Westminster Campus, 800 Commissioners Rd E, Room E2-646, London, Ontario, Canada, N6A 5W9; E-mail: drpower@powerplasticsurgery.com

Supported by the University of Western Ontario Academic Development Fund (Small Grants Competition) and the University of Western Ontario Clinician Investigator Program.

Presented at the 56th Annual Meeting of the Plastic Surgery Research Council, April 28-30, 2011, Louisville, KY, and the 69th Annual Meeting of the American Cleft Palate-Craniofacial Association, Paul Black Junior Investigator Session, April 17-21, 2012, San Jose, CA. The authors report no conflicts of interest.

Copyright $(\mathrm{C} 2014$ by Mutaz B. Habal, MD

ISSN: $1049-2275$

DOI: $10.1097 /$ SCS.0000000000001241
C ranial vault reconstruction may be indicated for patients with congenital and acquired skull defects. It is clinically relevant to understand how the skull heals full-thickness defects, both in adults and in children still undergoing brain growth. For reconstruction of craniosynostosis, segments from the cranial vault are removed, reoriented, and replaced as nonvascularized bone grafts in a more anatomic three-dimensional shape. Surgeons must expand the cranial vault and fossae using a finite quantity of bone, which may leave residual full-thickness defects to spontaneously heal. It is unknown why certain calvarial defects may close, whereas others persist or even increase in size (Fig. 1).

Osteogenic properties of the periosteum and dura have been confirmed. $^{1-8}$ It remains uncertain whether bone graft vascularity affects basic mechanisms of dural osteogenesis. Despite this incomplete understanding, the calvarial defect model has been applied within hundreds of basic science studies in the plastic surgery literature, which may confound results when studying the effectiveness of various bone substitutes.

The purpose of this study was to develop a model to study the effects of nonvascularized $(\mathrm{N}-\mathrm{V})$ versus vascularized (VAS) bone grafting on bone production within calvarial defects over time using microcomputed tomography (micro-CT). Additional objectives were (1) to compare patterns of bone healing between the groups and (2) to determine whether differences exist over time in mineralization of $\mathrm{N}-\mathrm{V}$ versus VAS grafts.

\section{MATERIALS AND METHODS}

Ethics approval was granted from the Council on Animal Care at our local institution (no. 2009-088). Adult male Wistar rats were randomly assigned to control $(n=6), N-V(n=10)$, and VAS $(\mathrm{n}=10)$ bone graft groups. The animals were 10 weeks of age and approximately $300 \mathrm{~g}$ at the time of surgery.

\section{Operative Technique}

Surgeries were performed under isoflurane anesthetic at $\times 2.5$ loupe magnification. A $3-\mathrm{cm}$ incision was made through the midline scalp, extending from the prefrontal to occipital region. Periosteal flaps were reflected laterally, exposing the denuded parietal bones.

Study design incorporated a bilateral model, providing a defect versus treatment (sham or bone graft) side within each animal. Calvarial defects and bone grafts were created using a hand-driven dermal biopsy punch according to surgical group. A dermal punch was used to decrease potential for thermal injury to bone. Procedures were limited to the parietal bones bilaterally, which excluded connective tissue of cranial sutures from all calvarial defects and bone grafts.

\section{Control Group $(n=6)$}

On the defect side (right parietal bone), a 4-mm calvarial defect was created, in addition to an adjacent full-thickness crescent defect. On the treatment (sham) side (left parietal), a single 4-mm 


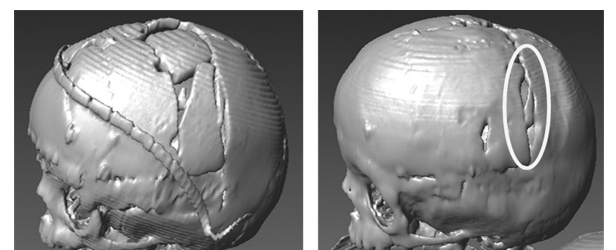

FIGURE 1. Computed tomographic scans of a child's skull after reconstruction at 1 day (left) and 1 year (right) postoperatively. Several calvarial defects have spontaneously healed, whereas others (circled) have increased in size in the parietal region.

calvarial defect was created. Native parietal bone surrounding the sham defect was not disrupted (Fig. 2A).

\section{N-V Group $(\mathbf{n}=\mathbf{1 0})$}

Four-millimeter parietal defects were created bilaterally. Within the left parietal bone (defect side), an adjacent fullthickness crescent defect was also left empty. On the bone graft side (the right parietal region), N-V bone grafting was performed. The $\mathrm{N}-\mathrm{V}$ bone grafts were completely incised along the margins and removed from the cranial vault, therefore disrupting the blood supply from dural arterioles. The crescent-shaped bone was then irrigated and returned to the harvest site as an N-V graft (Fig. 2B).

\section{VAS Group $(n=10)$}

As performed in the N-V group, 4-mm parietal defects were created bilaterally. An adjacent full-thickness crescent defect was also made in the left parietal bone (defect side). The bone graft side
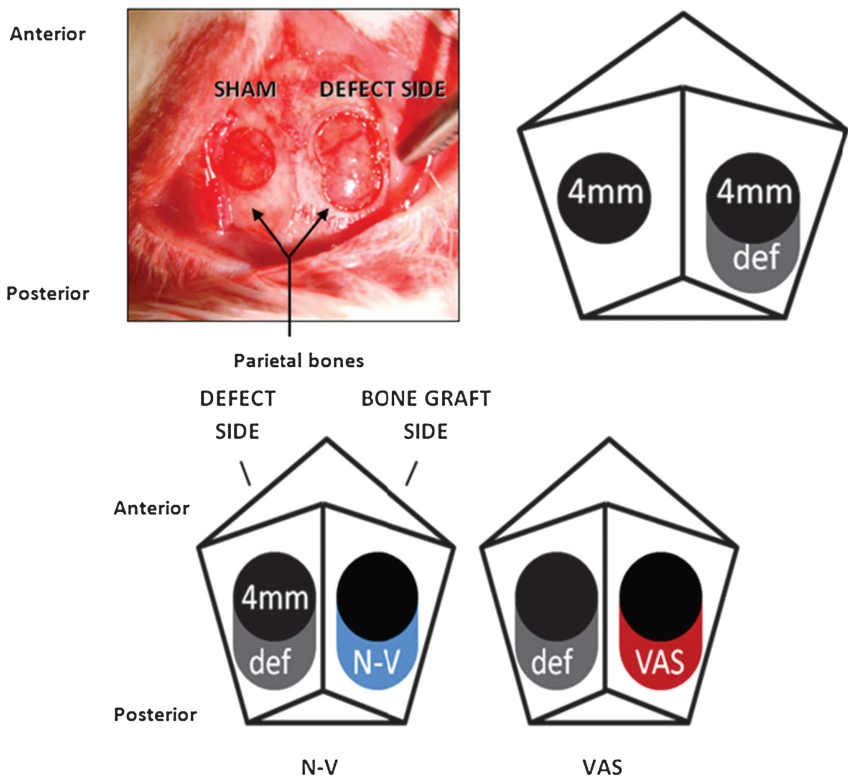

A $\quad(n=10) \quad B \quad(n=10)$

FIGURE 2. A, Control group $(n=6)$. The intraoperative photograph on the left shows a bird's-eye view of the rat skull. Anterior and posterior references are marked. Bilateral calvarial defects were created in the parietal bones. Dura and dural arterioles are visible at the base of the full-thickness defects. The schematic diagram corresponds to the intraoperative photograph. (4 mm indicates full-thickness calvarial defect with 4-mm diameter; def, full-thickness crescentic defect). B, The N-V and VAS groups showing defect and bone graft sides within the left and right parietal bones, respectively. Anterior and posterior references are marked. ( $4 \mathrm{~mm}$ indicates full-thickness calvarial defect with 4-mm diameter; def, full-thickness crescentic defect.) (the right parietal region) underwent VAS bone grafting adjacent to the 4-mm calvarial defect. The VAS bone grafts were completely incised along the margins until ballottable using the tip of the scalpel blade. They were not removed from the cranial vault however, which preserved vascularity from underlying dural arterioles (Fig. 2). Vascularity was confirmed through inspection intraoperatively.

Care was taken to avoid injury to the underlying dura and brain while creating calvarial defects and harvesting bone grafts. Malleable retractors were positioned to prevent contact between the dermal biopsy punch and dura at the crescent margin. Saline irrigation was performed intermittently to avoid desiccation of bone or dura.

Within all groups, two $5^{\prime} 0$ polyglactin sutures were secured between the temporalis muscles across the parietal bones. These sutures stabilized bone grafts in the N-V and VAS groups and prevented migration. Sutures were similarly placed in the control group for standardization purposes. The periosteum was then reapproximated, followed by skin closure.

\section{Imaging Protocol and Analysis}

Serial micro-CT scans were performed under isoflurane anesthetic at postoperative weeks 0, 2, 6, and 12 using the SpeCZT micro-CT scanner (GE Healthcare Biosciences, London, ON, Canada). The micro-CT scans were analyzed using MicroView software (Version 2.1.2; GE Healthcare Biosciences). Protocol for imaging acquisition and analysis has previously been described. ${ }^{9}$ Bone analysis was performed to calculate bone mineral content (BMC) within the calvarial defects and the bone grafts over time. Bone healing patterns were also recorded, either progressing as islands of new bone formation or limited to defect margins. Regions of interest (ROIs) were selected on baseline micro-CT scans and applied to coregistered scans over time (Figs. 3A, B).

\section{Statistical Analysis}

Statistical analysis was performed using SPSS software, version 17 (IBM, Somers, NY). Within the groups, BMC was compared between defect and treatment (sham or bone graft) sides at each time point using paired $t$-tests. Differences in BMC between treatment and defect (T-D) sides were compared between the groups using 1-way analysis of variance (ANOVA) at each time point. If a difference was found using ANOVA, post hoc comparisons were performed using the Tukey honestly significant difference $t$-test to identify the difference between pairs. Nonparametric analysis was

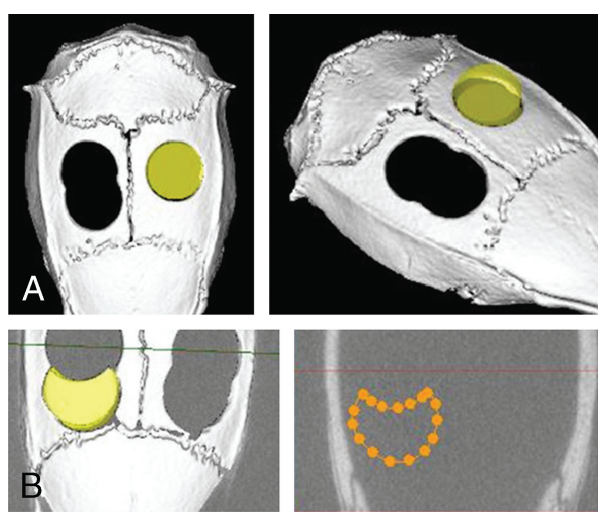

FIGURE 3. A, Example of cylindrical ROI created within a calvarial defect (bird's-eye and oblique views on left and right, respectively). B, Example of crescentic ROI created using spline tool in MicroView. 


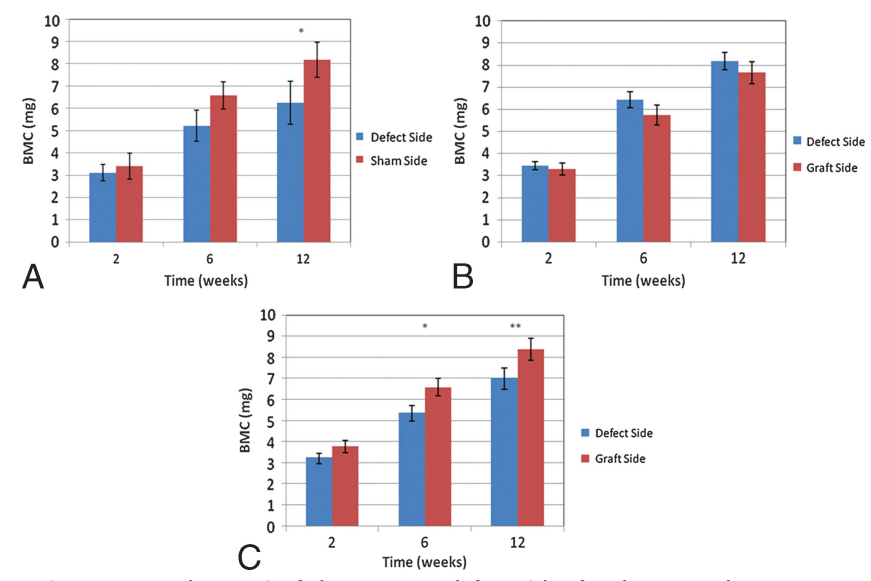

FIGURE 4. A, The BMC of sham versus defect sides for the control group. The BMC expressed in milligrams. Asterisk indicates $P=0.042$. B, The BMC of bone graft versus defect sides for the N-V group. The BMC expressed in milligrams. $C$, The BMC of bone graft versus defect sides for the VAS group. The BMC expressed in milligrams. Asterisk indicates $P=0.032$; double asterisk $P=0.024$. Error bars represent SEM

used to support findings from parametric tests due to small sample sizes. The Kruskal-Wallis and Wilcoxon 2-sample tests were applied for 3- and 2-group comparisons, respectively.

The BMCs of posterior crescents on the N-V versus VAS bone graft sides were compared using unpaired $t$-tests at each time point. Differences between T-D posterior crescents were also compared between the groups over time using unpaired $t$-tests. Crescent volume was compared between the groups using ANOVA to determine whether significant differences existed in the size of empty, $\mathrm{N}-\mathrm{V}$, and VAS bone graft crescents.

The McNemar test was applied within the groups to compare patterns of bone formation between the sides. The Fisher exact test was used to compare healing patterns between the groups.

Fourteen randomly selected CT scans were measured on 3 different days to calculate intrarater reliability. ${ }^{10}$ Repeat BMC measurements were compared using intraclass correlation coefficients.

\section{RESULTS}

Surgeries were performed on 26 male Wistar rats ( 6 controls, 10 per bone graft group). Intraoperatively, the VAS bone grafts demonstrated a pink cast similar to adjacent bone, which suggested that vascularity was preserved from perforating dural arterioles. In contrast, the N-V bone grafts appeared white. No postoperative complications were encountered. Micro-CT scans taken at week 0 did not reveal any subdural or epidural collections or pneumocephalus.

Conclusions from parametric tests were confirmed using the nonparametric analysis. The $P$ values within this section refer to the parametric analysis. No differences in ROI volume were seen

TABLE 1. Parametric Analysis Within Groups

\begin{tabular}{llll}
\hline Group & Week 2 & Week 6 & Week 12 \\
\hline Control & 0.550 & 0.091 & $0.042^{*}$ \\
N-V & 0.597 & 0.193 & 0.427 \\
VAS & 0.079 & $0.032 *$ & $0.024^{*}$
\end{tabular}

Paired $t$-tests comparing BMC of treatment (sham or bone graft) versus defect sides within the groups over time.

*Significance, $P<0.05$
TABLE 2. Nonparametric Analysis Within Groups

\begin{tabular}{llll}
\hline Group & Week 2 & Week 6 & Week 12 \\
\hline Control & 0.917 & $0.028^{*}$ & $0.028^{*}$ \\
N-V & 0.508 & 0.169 & 0.333 \\
VAS & 0.074 & $0.028^{*}$ & $0.037^{*}$ \\
\hline
\end{tabular}

Wilcoxon 2-sample tests comparing BMC of treatment (sham or bone graft) versus defect sides within the groups over time.

*Significance, $P<0.05$.

between the groups for empty, N-V, and VAS crescents. Intrarater reliability demonstrated an intraclass correlation coefficient of 0.928 .

\section{Bone Mineral Content: Treatment Versus Defect Sides Within the Groups}

The control group demonstrated greater BMC on the sham versus defect side at week $12(P=0.042$; Fig. 4A, Tables 1 and 2$)$. No differences existed in the BMC on the sham versus defect sides within the control group at other time points.

Within the N-V group, the BMC between the sides was not statistically different over time (Fig. 4B, Tables 1 and 2). Greater BMC was seen within the VAS group on the bone graft versus defect side at weeks $6(P=0.032)$ and $12(P=0.024$; Fig. $4 \mathrm{C}$, Tables 1 and 2$)$.

\section{Bone Mineral Content: Treatment Minus Defect Sides Between the Groups}

Differences in BMC between treatment (sham or bone graft) and defect (T-D) sides for all groups over time are shown in Figure 5. The T-D differences were significant between the groups at weeks $6(P=0.016)$ and $12(P=0.025$; Table 3$)$. At week 6 , the T-D difference was greater for the control versus the $\mathrm{N}-\mathrm{V}$ group $(P=0.043$; Table 4). The T-D difference was also greater for the VAS versus N-V group $(P=0.030)$. No differences were seen between the control and VAS groups $(P=0.978)$ at week 6 . At week 12, T-D differences were also greater for the control and VAS versus $\mathrm{N}-\mathrm{V}$ groups $(P=0.038$ and 0.050 , respectively). The control and VAS groups did not show any differences $(P=0.817)$.

\section{Bone Mineral Content: Posterior Crescents in the N-V Versus VAS Groups}

At week 2, BMC was higher for posterior crescents in the VAS versus N-V groups on the bone graft side $(P=0.007$, Table 5). No differences in the posterior crescent BMC were seen between the N-V and VAS groups at week 6 or $12(P=0.386$ and 0.645 , respectively).

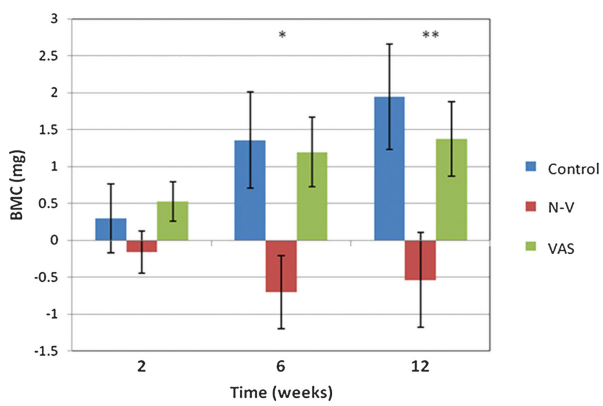

FIGURE 5. The BMC differences for treatment minus defect (T-D) sides within the groups over time. The BMC expressed in milligrams. Error bars represent SEM. Asterisk indicates $P=0.016$; double asterisk, $P=0.025$. 
TABLE 3. BMC Differences Between Groups

\begin{tabular}{lrc}
\hline Week & \multicolumn{1}{c}{ ANOVA } & Kruskal-Wallis \\
\hline 0 & $P=0.515$ & 0.869 \\
2 & 0.278 & 0.140 \\
6 & $0.016^{*}$ & $0.046^{*}$ \\
12 & $0.025^{*}$ & 0.077 \\
\hline
\end{tabular}

Statistical comparisons of BMC differences for treatment minus defect (T-D) sides between the groups.

* Significance, $P<0.05$.

The BMC differences for treatment minus defect (T-D) posterior crescents for the N-V and VAS groups over time are shown in Figure 6. The T-D posterior crescent differences were greater within the VAS versus N-V group at week $2(P=0.050$; Table 6). Differences for T-D posterior crescents were no longer significant between the N-V and VAS groups at week $6(P=0.423)$ or 12 $(P=0.467)$.

\section{Patterns of Bone Formation}

Bone healing patterns for defect and treatment (sham or bone graft) sides within the groups are included in Figures 7A and B, respectively. In the control group, healing progressed through islands of bone formation within $50 \%$ of defect sides and the majority $(83.3 \%)$ of sham sides. Most defect sides $(70 \%)$ in the N-V group healed through islands of new bone. The N-V bone graft sides, however, healed primarily from bone margins $(70 \%)$. In the VAS group, bone healing occurred through islands of bone formation within the majority of defect $(70 \%)$ and treatment $(80 \%)$ sides.

Within the groups, bone healing patterns were not statistically different between the sides (control, $P=0.500 ; \mathrm{N}-\mathrm{V}, P=0.289$; VAS, $P=1.000)$. Across the group, comparisons did not show healing pattern differences on the defect side $(P=0.758)$. Healing patterns on the treatment (sham or bone graft) side were different, however, between the control, $\mathrm{N}-\mathrm{V}$, and VAS groups $(P=0.047)$. In the control and VAS groups, healing occurred mostly as islands of new bone formation when framed by VAS bone (Figs. 7C and D, respectively). When partially filled with N-V bone grafts, healing occurred mostly at defect margins in the $\mathrm{N}-\mathrm{V}$ group (Fig. 7E).

\section{DISCUSSION}

Previous research on calvarial defect healing has largely focused on the effectiveness of scaffolds and bone substitutes. ${ }^{1-20}$ Further studies have evaluated the osteogenic potential of local factors within bone graft recipient sites, including the dura, periosteum, and adjacent bone. The dura is believed to be the dominant factor promoting revascularization and bone healing. ${ }^{4,21}$ Limited osteogenic and angiogenic properties have been shown for adjacent bone when bone graft healing is isolated from the dura and

TABLE 4. Post Hoc Analysis of BMC Differences Using 2-Group Comparisons

\begin{tabular}{lll}
\hline Group Comparisons & Week 6 & Week 12 \\
\hline Control vs N-V & $0.043^{*}$ & $0.038^{*}$ \\
Control vs VAS & 0.978 & 0.817 \\
N-V vs VAS & $0.030^{*}$ & $0.050^{*}$ \\
\hline
\end{tabular}

Post hoc Tukey honestly significant difference test to identify differences between the 2-group comparisons after 1-way ANOVA.

*Significance, $P<0.05$.
TABLE 5. Analysis of Graft BMC Over Time: VAS versus N-V

\begin{tabular}{lcccc}
\hline Comparison & Week 0 & Week 2 & Week 6 & Week 12 \\
\hline$t$-test & 0.117 & $0.007^{*}$ & 0.386 & 0.645 \\
Wilcoxon & 0.123 & $0.009^{*}$ & 0.529 & 0.796 \\
\hline \multicolumn{2}{r}{ Statistical comparisons for posterior crescents on the treatment side between N-V } \\
and VAS over time. \\
$\quad$ *Significance, $P<0.05$. \\
$\quad t$-Test, unpaired $t$-tests. Wilcoxon, Wilcoxon 2-sample tests.
\end{tabular}

periosteum. ${ }^{4}$ The dura is theorized to be actively osteogenic as well as promoting adjacent calvarial marrow to form bone through production of growth factors. ${ }^{4,21}$ Recipient sites in these studies contained $\mathrm{N}-\mathrm{V}$ bone grafts, which provided an acellular scaffold for bone healing. ${ }^{4,21}$

Cranial vault surgery may incorporate both N-V and VAS bone segments during reconstruction. The effects of bone graft vascularity on basic mechanisms of calvarial healing remain unknown. The calvarial defect model established within this study maintained intact dura at the calvarial defect base and reapproximated the periosteum. Surgical technique created calvarial defects and bone grafts using a hand-driven dermal biopsy punch to minimize potential for thermal injury to bone. ${ }^{22}$ Powered instruments may lead to detrimental effects on the dura, blood vessels, and adjacent bone through heat production, which may confound results. ${ }^{23}$

Surgical groups differed only with respect to the adjacent bone crescent on the sham or bone graft side, including native parietal bone, N-V, or VAS bone graft. Pericranial vessels were disrupted in all groups during parietal bone exposure. In the control group, the adjacent crescent was supplied by the arterial network within the parietal bone, in addition to perforating arterioles from the dura. The VAS bone grafts were supplied by perforating dural arterioles. For the N-V bone grafts, the parietal bone arterial plexus, dural, and pericranial vessels had all been disrupted.

Findings suggest that bone graft vascularity may significantly affect the quantity of bone production during calvarial healing. At weeks 6 and 12, healing was impaired on the $\mathrm{N}-\mathrm{V}$ bone graft side versus the VAS and sham sides. Calvarial healing was not statistically different in the presence of native parietal bone versus VAS bone grafts. Nonvascularized bone grafts may initiate greater osteoclastic activity, which may decrease overall bone production during calvarial healing. An alternative theory is that dural osteogenesis may be inhibited by local release of intracellular contents after osteocyte death in $\mathrm{N}-\mathrm{V}$ bone grafts. Revascularization of $\mathrm{N}-\mathrm{V}$ grafts may be incomplete, which may make delivery of dural growth factors less efficient as well as result in fewer endothelial cells to stimulate.

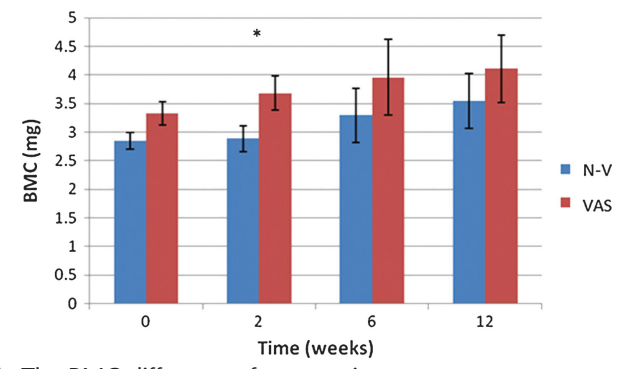

FIGURE 6. The BMC differences for posterior crescents on treatment (bone graft) minus defect (T-D) sides for the N-V and VAS groups over time. The BMC differences expressed in milligrams. Error bars represent SEM. Asterisk indicates $P=0.050$. 
TABLE 6. BMC Differences Between Sides Across Groups: VAS versus N-V

\begin{tabular}{lclcc}
\hline Comparison & Week 0 & Week 2 & Week 6 & Week 12 \\
\hline$t$-test & 0.072 & $0.050^{*}$ & 0.423 & 0.467 \\
Wilcoxon & 0.052 & 0.063 & 0.529 & 0.529
\end{tabular}

Comparisons of BMC differences in posterior crescents on treatment minus defect (T-D) sides between $\mathrm{N}-\mathrm{V}$ and VAS over time.

* Significance, $P<0.05$.

$t$-Test, unpaired $t$-tests. Wilcoxon, Wilcoxon 2-sample tests.

\section{Patterns of Calvarial Healing}

Heine (1926) observed that calvarial defect healing progressed from both the native bone edges and bone islands originating from dura mater. ${ }^{24}$ Dural osteogenesis is the dominant mechanism of calvarial healing, and bone formation decreases significantly in its absence. ${ }^{25,26}$ Bone healing progressed through islands of new bone formation within the majority of defect and treatment sides in the control and VAS groups in the presence of either native parietal bone or VAS bone grafts. In contrast, healing occurred primarily from bone margins on the $\mathrm{N}-\mathrm{V}$ bone graft side.

The majority of previous studies noted progression of calvarial healing from defect margins. Gurevitch et $\mathrm{al}^{27}$ observed healing from bone margins within calvarial defects filled with demineralized bone matrix. When fibrin glue was added, containing fibrinogen and thrombin, which are involved in the coagulation cascade, healing occurred as islands of new bone formation. Differences in healing patterns within previous studies may be attributed to the presence of inert scaffolds, as seen in the N-V group within this study. ${ }^{27-34}$ Sampling error of histologic preparations may be a confounding variable, however, in some cases. Histologic examinations are limited to two-dimensional slices, which may fail to identify small islands of bone formation. Bone islands were identified within this study using micro-CT, which provided high-resolution nondestructive imaging of three-dimensional bone architecture.

Bone graft vascularity may alter basic mechanisms of calvarial healing. Findings from this study suggest that a feedback loop may exist during calvarial healing, which determines whether appositional, dural, or pericranial osteogenesis is the dominant mechanism. In the presence of $\mathrm{N}-\mathrm{V}$ bone grafts, healing was limited to defect margins and seemed to inhibit dural osteogenesis. Nonvascularized grafts may shift the balance toward greater osteoclastic versus osteoblastic activity. Remodeling and revascularization of $\mathrm{N}-\mathrm{V}$ bone grafts may become the dominant process, making dural osteogenesis less efficient for repair of residual calvarial defects. Marginal healing seemed to progress through appositional bone growth.

Interestingly, calvarial healing was not statistically different between the defect versus bone graft sides within the N-V group. No differences in BMC were seen over time within the defects partially closed with $\mathrm{N}-\mathrm{V}$ bone graft versus those left empty, leaving an initial bone defect of larger surface area. The N-V bone graft crescents demonstrated greater BMC over time as compared with empty crescents and may provide a scaffold for calvarial healing. Although the N-V grafts resulted in greater healing at defect margins, they did not promote bone production within central defects.

\section{Calvarial Bone Graft Healing and Vascularity}

Fukuta et $\mathrm{al}^{35}$ found greater resorption of $\mathrm{N}-\mathrm{V}$ versus VAS bone grafts when placed as onlay grafts in the temporal fossa using water displacement measurements. The VAS bone grafts were supplied by pericranial vessels in contrast to the dural arteriolar supply to the VAS grafts within this study. Normal bone architecture was seen on histologic examination of VAS bone grafts at 12 weeks in comparison with fibrous tissue ingrowth within N-V grafts. ${ }^{35}$ Cutting and McCarthy ${ }^{36}$ similarly found less resorption of VAS versus $\mathrm{N}-\mathrm{V}$ onlay bone grafts in composite myo-osseous flaps assessed by bone sample weights.

Micro-CT permitted precise quantification of BMC within bone grafts over time within this study. No significant resorption occurred for either $\mathrm{N}-\mathrm{V}$ or VAS bone grafts placed as inlay grafts within parietal defects. The VAS bone grafts demonstrated greater BMC than the $\mathrm{N}-\mathrm{V}$ grafts did at 2 weeks postoperatively, which may suggest that vascularity plays a role in early bone graft healing. Previous studies have shown acellular marrow and empty lacunae in early N-V bone graft healing compared with osteocytes and cellular marrow within VAS grafts. ${ }^{37}$ Our results did not show any differences in BMC between the N-V and VAS bone grafts as healing progressed through 6 and 12 weeks postoperatively. The N-V bone grafts, although acellular, may provide a scaffold for early bone healing and ingrowth of blood vessels.

Effects of radiation on calvarial defect healing within this study remain unknown. The imaging protocol at our center has been optimized at approximately 30 cGy per acquisition to minimize radiation exposure while maintaining adequate resolution to study bone microarchitecture. ${ }^{38}$ All rats were subject to an equivalent cumulative dose. Both VAS and N-V bone grafts may potentially be affected by high-dose irradiation because of direct damage to microvessels and disruption of angiogenesis. ${ }^{39,40}$

Although VAS bone grafting was associated with enhanced dural osteogenesis, the mechanism of action was not proven by this study design. Biomolecular and histologic studies will be performed to measure osteoclastic versus osteoblastic activity to further understand mechanisms regulating dural osteogenesis.

In conclusion, a bilateral defect model was established to study the effects of $\mathrm{N}-\mathrm{V}$ versus VAS bone grafting on calvarial healing. Greater bone production occurred within native parietal bone and VAS bone grafts at later stages, and dural regeneration seemed to be primarily responsible for calvarial healing. The $\mathrm{N}-\mathrm{V}$ bone graft healing was restricted to defect margins. Greater understanding of the effects of calvarial vascularity on bone healing is needed before further application of the calvarial defect model within basic science studies.

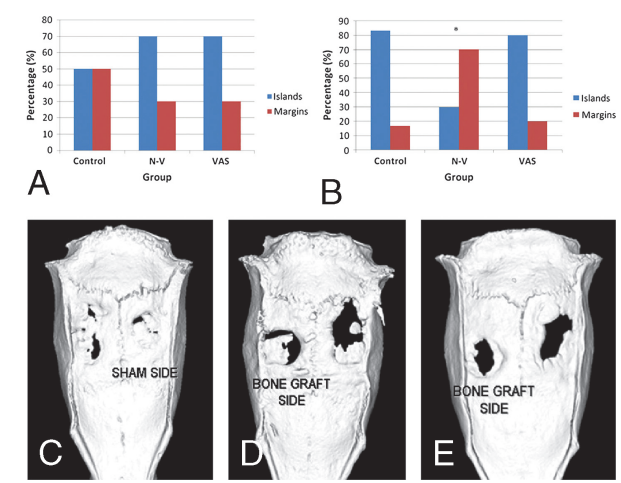

FIGURE 7. A, Patterns of bone formation on the defect side by group. $B$, Patterns of bone formation on the treatment (sham or bone graft) side by group. Difference between the groups in healing patterns on the treatment side is shown using ANOVA. Asterisk indicates $P=0.047$. C, Representative isosurface image for a control rat at week 12, showing healing through new bone islands on the sham (animal's left) side. D, Representative isosurface image for a VAS rat at week 12, showing healing primarily through islands of new bone formation on the bone graft (animal's right) side. E, Representative isosurface image for an N-V rat at week 12, showing marginal healing on the bone graft (animal's right) side. 


\section{ACKNOWLEDGMENTS}

The authors thank Larry Stitt for his assistance with statistical analysis as well as Steven Pollmann and Matt Teeter for their technical support.

\section{REFERENCES}

1. Kanou M, Ueno T, Kagawa T, et al. Osteogenic potential of primed periosteum graft in the rat calvarial model. Ann Plast Surg 2005;54: 71-78

2. Inoda H, Yamamoto G, Hattori T. Histological investigation of osteoinductive properties of rh-BMP2 in a rat calvarial bone defect model. J Craniomaxillofac Surg 2004;32:365-369

3. Honma T, Itagaki T, Nakamura M, et al. Bone formation in rat calvaria ceases within a limited period regardless of completion of defect repair. Oral Dis 2008;14:457-464

4. Ozerdem OR, Anlatici R, Bahar T, et al. Roles of periosteum, dura, and adjacent bone on healing of cranial osteonecrosis. J Craniofac Surg 2003;14:371-379

5. Breitbart AS, Grande DA, Kessler R, et al. Tissue engineered bone repair of calvarial defects using cultured periosteal cells. Plast Reconstr Surg 1998;101:567-574

6. Petrie Aronin CE, Cooper JA Jr, Sefcik LS, et al. Osteogenic differentiation of dura mater stem cells cultured in vitro on three-dimensional porous scaffolds of poly(epsilon-caprolactone) fabricated via co-extrusion and gas foaming. Acta Biomater 2008;4:1187-1197

7. Park JW, Jang JH, Bae SR, et al. Bone formation with various bone graf substitutes in critical-sized rat calvarial defect. Clin Oral Implants Res 2009;20:372-378

8. Kalish BP, Schuster GS, Peacock ME, et al. Influence of matrix-suspended demineralized bone on osseous repair using a critical-sized defect in the rat (Rattus norvegicus) calvarium. J Oral Implantol 2008;34:83-89

9. Power SM, Matic DB, Holdsworth DW. Accounting for cranial vault growth in experimental design. Oral Surg Oral Med Oral Pathol Oral Radiol 2014;117:e325-329

10. Gluer CC, Blake G, Lu Y, et al. Accurate assessment of precision errors: how to measure the reproducibility of bone densitometry techniques. Osteoporos Int 1995;5:262-270

11. Sikavitsas VI, van den Dolder J, Bancroft GN, et al. Influence of the in vitro culture period on the in vivo performance of cell/titanium bone tissue-engineered constructs using a rat cranial critical size defect model. J Biomed Mater Res A 2003;67:944-951

12. Bolgen N, Vargel I, Korkusuz P, et al. Tissue responses to novel tissue engineering biodegradable cryogel scaffolds: an animal model. J Biomed Mater Res A 2009;91:60-68

13. Burdick JA, Frankel D, Dernell WS, et al. An initial investigation of photocurable three-dimensional lactic acid based scaffolds in a critical-sized cranial defect. Biomaterials 2003;24: $1613-1620$

14. Castano-Izquierdo H, Alvarez-Barreto J, van den Dolder J, et al. Pre-culture period of mesenchymal stem cells in osteogenic media influences their in vivo bone forming potential. $J$ Biomed Mater Res A 2007;82:129-138

15. Cowan CM, Aghaloo T, Chou YF, et al. MicroCT evaluation of three-dimensional mineralization in response to BMP-2 doses in vitro and in critical sized rat calvarial defects. Tissue Eng 2007;13:501-512

16. Petrie Aronin CE, Sadik KW, Lay AL, et al. Comparative effects of scaffold pore size, pore volume, and total void volume on cranial bone healing patterns using microsphere-based scaffolds.

J Biomed Mater Res A 2009;89:632-641

17. Woodruff MA, Rath SN, Susanto E, et al. Sustained release and osteogenic potential of heparan sulfate-doped fibrin glue scaffolds within a rat cranial model. J Mol Histol 2007;38:425-433

18. Sawyer AA, Song SJ, Susanto E, et al. The stimulation of healing within a rat calvarial defect by $\mathrm{mPCL}-\mathrm{TCP} /$ collagen scaffolds loaded with rhBMP-2. Biomaterials 2009;30:2479-2488
19. Osathanon T, Linnes ML, Rajachar RM, et al. Microporous nanofibrous fibrin-based scaffolds for bone tissue engineering. Biomaterials 2008;29:4091-4099

20. Montjovent MO, Mathieu L, Schmoekel H, et al. Repair of critical size defects in the rat cranium using ceramic-reinforced PLA scaffolds obtained by supercritical gas foaming. J Biomed Mater Res A 2007;83:41-51

21. Hopper RA, Zhang JR, Fourasier VL, et al. Effect of isolation of periosteum and dura on the healing of rabbit calvarial inlay bone grafts. Plast Reconstr Surg 2001;107:454-462

22. Eriksson A, Albrektsson T, Grane B, et al. Thermal injury to bone. A vital-microscopic description of heat effects. Int J Oral Surg 1982;11:115-121

23. Cooper GM, Mooney MP, Gosain AK, et al. Testing the critical size in calvarial bone defects: revisiting the concept of a critical-size defect. Plast Reconstr Surg 2010;125:1685-1692

24. Vogeler K, Redenz E, Walter H, et al. Bernhard Heine's Versuche über Knochenregeneration: Sein Leben und seine Zeit. Berlin: Verlag von Julis Springer, 1926

25. Mossaz CF, Kokich VG. Redevelopment of the calvaria after partial craniectomy in growing rabbits: the effect of altering dural continuity. Acta Anat (Basel) 1981;109:321-331

26. Moss ML. Growth of the calvaria in the rat; the determination of osseous morphology. Am J Anat 1954;94:333-361

27. Gurevitch O, Kurkalli BG, Prigozhina T, et al. Reconstruction of cartilage, bone, and hematopoietic microenvironment with demineralized bone matrix and bone marrow cells. Stem Cells 2003;21:588-597

28. Develioglu H, Unver Saraydin S, Kartal U. The bone-healing effect of a xenograft in a rat calvarial defect model. Dent Mater J 2009; 28:396-400

29. Moon HJ, Kim KN, Kim KM, et al. Effect of calcium phosphate glass on bone formation in calvarial defects of Sprague-Dawley rats. $J$ Mater Sci Mater Med 2006;17:807-813

30. Moon HJ, Kim KN, Kim KM, et al. Bone formation in calvarial defects of Sprague-Dawley rats by transplantation of calcium phosphate glass. J Biomed Mater Res A 2005;74:497-502

31. Artzi Z, Kozlovsky A, Nemcovsky CE, et al. Histomorphometric evaluation of natural mineral combined with a synthetic cell-binding peptide (P-15) in critical-size defects in the rat calvaria. Int J Oral Maxillofac Implants 2008;23:1063-1070

32. Gomez G, Korkiakoski S, Gonzalez MM, et al. Effect of FGF and polylactide scaffolds on calvarial bone healing with growth factor on biodegradable polymer scaffolds. J Craniofac Surg 2006;17:935-942

33. Develioglu H, Saraydin SU, Bolayir G, et al. Assessment of the effect of a biphasic ceramic on bone response in a rat calvarial defect model. J Biomed Mater Res A 2006;77:627-631

34. Schliephake H, Tavassol F, Gelinsky M, et al. Use of a mineralized collagen membrane to enhance repair of calvarial defects in rats. Clin Oral Implants Res 2004;15:112-118

35. Fukuta K, Har-Shai Y, Collares MV, et al. The viability of revascularized calvarial bone graft in a pig model. Ann Plast Surg 1992;29:136-142

36. Cutting CB, McCarthy JG. Comparison of residual osseous mass between vascularized and nonvascularized onlay bone transfers. Plast Reconstr Surg 1983;72:672-675

37. Antonyshyn O, Colcleugh RG, Hurst LN, et al. The temporalis myo-osseous flap: an experimental study. Plast Reconstr Surg 1986;77:406-415

38. Umoh JU, Sampaio AV, Welch I, et al. In vivo micro-CT analysis of bone remodeling in a rat calvarial defect model. Phys Med Biol 2009;54:2147-2161

39. Langley RE, Bump EA, Quartuccio SG, et al. Radiation-induced apoptosis in microvascular endothelial cells. Br J Cancer 1997;75: 666-672

40. Okunieff P, Wang X, Rubin P, et al. Radiation-induced changes in bone perfusion and angiogenesis. Int J Radiat Oncol Biol Phys 1998;42: $885-889$ 